
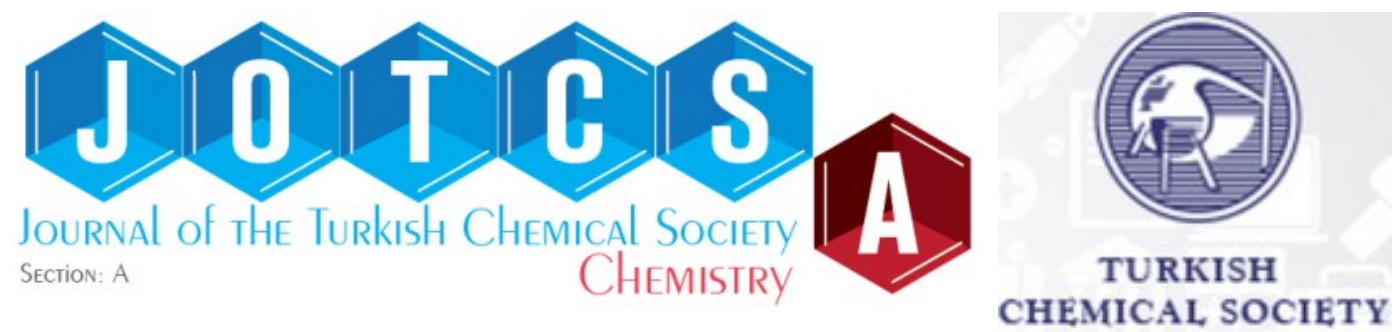

\title{
Laccase-catalyzed Conjugation of BSA Mediated by Gallic Acid: Preparation, Characterization, and Antioxidant Activity
}

\author{
Merve Bat-Özmatara1 $\triangle$ iD, Fatma Ertan'1 \\ ${ }^{1}$ Gebze Technical University, Department of Chemistry, Gebze- Kocaeli, Turkey.
}

\begin{abstract}
Laccase is one of the enzymes that catalyze the oxidation of phenolic and non-phenolic substrates and show encouraging potential as a biocatalyst in the synthesis of bioactive compounds. It is known that phenolic acids have an antioxidant effect. Bovine serum albumin (BSA) shows gelling activity, and nutraceutical binding ability but it does not show antioxidant activity. In this study, BSA which has no antioxidant activity using laccase, started to show antioxidant activity with gallic acid (GA) conjugation. The synthesized conjugates were analyzed by polyacrylamide gel electrophoresis (PAGE), ultraviolet-visible spectrophotometry (UV-Vis), and Fourier-transform infrared spectroscopy (FTIR). Radical scavenging capacity for antioxidant activity was measured. GA-functionalized-BSA displayed greatly improved 2,2'-azinobis-(3- ethylbenzothiazoline-6-sulfonic acid) (ABTS) and 1,1-diphenyl-2picryl-hydrazyl (DPPH) radical scavenging capacities, compared with the untreated BSA. Proteinflavonoid conjugates can improve the natural properties of proteins, being promising products to be used in medical, food and polymer fields where antioxidant ability is an essential feature.
\end{abstract}

Keywords: Laccase, bioconjugate, BSA, gallic acid, antioxidant activity.

Submitted: June 14, 2021. Accepted: November 29, 2021.

Cite this: Bat-Özmatara M, Ertan F. Laccase-catalyzed Conjugation of BSA Mediated by Gallic Acid: Preparation, Characterization, and Antioxidant Activity. JOTCSA. 2022;9(1):29-36.

DOI: https://doi.org/10.18596/jotcsa.952065.

*Corresponding author. E-mail: mervebatt@gmail.com.

\section{INTRODUCTION}

It is well known that phenolic compounds exhibit antioxidant and antimicrobial activity and have health benefits. Phenols in foods have been shown to have many biological functions such as anticarcinogens, anti-aging, antimutagenes, antidiabetic and immunostimulatory effects, leading to their recognition as potential nutraceuticals $(1,2)$. Phenolic compounds are simple and naturally occurring compounds containing one or more linked phenolic rings. Various natural phenolic compounds such as gallic acid, hydroxycinnamic acid, hydroxybenzoic acid, ferulic acid, caffeic, p-coumaric acid, vanillin, benzoic acid, catechols are seen in fruits, vegetables and cereals (3). Gallic acid (GA) (3,4,5trihydroxybenzoic acid), is a powerful antioxidant that protects against oxidative damage caused by reagents such as hydroxyl $(\mathrm{HO} \cdot)$, superoxide $\left(\mathrm{O}_{2}{ }^{-}\right)$ and peroxyl ( $\mathrm{ROO}{ }^{\circ}$ ) hydrogen peroxide $\left(\mathrm{H}_{2} \mathrm{O}_{2}\right)$ and hypochlorous acid ( $\mathrm{HOCl})(4)$. There are studies in the literature showing that gallic acid has antioxidant and anticancer properties $(5,6)$.

Laccase (benzenediol: oxygen oxidoreductases, EC 1.10.3.2) catalyzes the direct oxidation of an aromatic substrate in the presence of molecular oxygen, which is reduced to water during the reaction. This reaction involves the four singleelectron oxidation of the reducing substrate coupled to the four electron reductive cleavage of a dioxygen bond using four $\mathrm{Cu}$ atoms distributed between three regions (7). The laccase enzyme has an ability to catalyze a wide variety of substrates such as phenols, aromatic, aliphatic amines, and their derivatives. Laccase was used in the enzymatic polymerization of catechol $(8,9)$. Laccase catalyzed crosslinking of a-lactalbumin and ferulic acid was investigated (10). Some 
studies show that phenolic substrates such as caffeic acid, ferulic acid, vanillic acid, gallate, and p-coumaric acid can be used in laccase catalyzed crosslinking of proteins $(11,12)$.

Bovine serum albumin (BSA or "Fraction V") is a serum albumin protein derived from cows. Serum albumin, also known as albumin for short, is the most common protein found in the blood plasma of humans and other mammals. It makes up $60 \%$ of the proteins in the blood. It is also found in tissue fluids, particularly muscle and skin, and small amounts of tears, sweat, gastric juices, and bile. $30-40 \%$ of the total albumin in the body is in the blood. In addition to carrying fatty acids and various other substances in the blood, its most important function is to balance water between blood and tissue fluids. Bovine serum albumin (BSA) shows gelling activity, surface activity, and nutraceutical binding ability but it does not show antioxidant activity $(13,14)$. In the literature, chitosan and dextran conjugates were made with laccase enzyme $(15,16,17)$. However, no laccase mediated BSA-gallic acid conjugate has been reported.

In this study, the BSA molecule, which has various physiological functions such as contributing to osmotic blood pressure, drug, and other molecule carriers, gained antioxidant activity by conjugating with gallic acid under the effect of laccase.

\section{EXPERIMENTAL SECTION}

\section{Materials}

DPPH, ABTS, Trizma, acrylamide, ammonium persulfate, glycerol, potassium persulfate $\left(\mathrm{K}_{2} \mathrm{~S}_{2} \mathrm{O}_{8}\right)$ and laccase from Trametes versicolor were purchased from Sigma. Ethanol, methanol, sodium dodecyl sulfate (SDS), bromophenol blue, 2mercaptoethanol, Coomassie brilliant blue R250, acetic acid, and sodium acetate were purchased from Merck. All solvents were of analytical grade.

\section{Laccase activity}

The laccase enzyme activity was spectrophotometrically measured by measuring the enzymatic oxidation of $0.5 \mathrm{mM}$ ABTS at $420 \mathrm{~nm}$. Molar extinction coefficient for the oxidation product was $3.6 \times 10^{4} \mathrm{~cm}^{-1} \mathrm{M}^{-1}$. A unit laccase (U) defined as the amount of enzyme required to oxidize $1 \mathrm{~mol}$ of ABTS per minute at room temperature (18).

\section{Preparation of acid-protein conjugates}

BSA and gallic acid solutions were prepared in 10 mM 4-(2- hydroxyethyl)-1 piperazineethanesulfonic acid (HEPES) buffer solution, $\mathrm{pH} 7.4$ with protein concentration at $5 \mathrm{mg} \mathrm{mL}^{-1}$. The oxidation reaction was initiated by adding laccase. The sample with 2 different enzyme amounts, $5 \mu \mathrm{L}$ and $15 \mu \mathrm{L}$, was prepared from $11 \mathrm{U} / \mathrm{L}$ enzyme. It was left in the shaking incubation at $50{ }^{\circ} \mathrm{C}$ for 24 hours (19).

\section{UV spectroscopy}

BSA, gallic acid and conjugates were monitored with a UV-Vis spectrophotometer (SpectraMax Plus 384 Microplate Reader, California, USA). Spectra were collected at room temperature before and after adding laccase.

\section{FTIR spectroscopy}

FT-IR analysis was performed on the BSA, gallic acid, and the conjugate were recorded using the Perkin Elmer FTIR spectrometer. The transmittance values were measured within the spectra between 4000 and $600 \mathrm{~cm}^{-1}$.

\section{SDS PAGE}

The molecular weight distribution of BSA, gallic acid and BSA-gallic acid conjugate were analyzed by SDS-PAGE which was carried out on $5 \%$ stacking gel and $12 \%$ separating gel. The $30 \mu \mathrm{L}$ of sample was mixed with $10 \mu \mathrm{L}$ of $4 \times$ sample buffer and then heated at $100{ }^{\circ} \mathrm{C}$ for $15 \mathrm{~min} .10 \mu \mathrm{L}$ of samples were loaded onto the wells and SDS-PAGE was operated at 120 volts. After electrophoresis, Coomassie Bright Blue R250 was adapted to gel staining for thirty minutes followed by decolorization with water overnight.

\section{Antioxidant Activity}

$D P P H$ radical scavenging activity

DPPH free radical scavenging activity was measured to evaluate the antioxidant capacity. The Brand-Williams method was used to test whether the sample bleached the stable DPPH radical (20). For this, $0.75 \mathrm{~mL}$ of plant extract was added onto $1.50 \mathrm{~mL}$ of DPPH solution prepared in ethanol (0.05 $\mathrm{mM})$. After shaking vigorously, the mixture was kept at room temperature for 30 minutes. After 30 minutes, absorbance at $517 \mathrm{~nm}$ was measured in a UV-Vis spectrophotometer. The scavenging activity of the DPPH radical was calculated using the formula:

DPPH scavenging $(\%)=\left[\left(A_{\text {control }}-A_{\text {sample }}\right) / A_{\text {control }}\right] x$ 100 (Eq. 1)

Herein, Acontrol shows the absorbance of the control (DPPH solution without sample) and $A_{\text {sample shows }}$ the absorbance of the test sample.

\section{ABTS radical scavenging activity}

This method, developed by Arnao et al., is based on reducing the cationic radical formed by the $\mathrm{K}_{2} \mathrm{~S}_{2} \mathrm{O}_{8}$ oxidation of ABTS with antioxidants. The blue / green colored ABTS •+ radical has a strong absorption at $600-750 \mathrm{~nm}(21)$.

For ABTS radical scavenging activity, the $7.4 \mathrm{mM}$ ABTS solution and $2.6 \mathrm{mM}$ potassium persulfate solution were evenly mixed, then kept in the dark 
at room temperature for 12 hours. 1 milliliter of the formed ABTS radical solution was diluted with an absorbance of $1.1 \pm 0.02$ at $734 \mathrm{~nm}$ wavelength in the spectrophotometer by adding approximately $60 \mathrm{~mL}$ of methanol. Then, $150 \mu \mathrm{L}$ of sample solution and $2850 \mu \mathrm{L}$ of ABTS ${ }^{\bullet+}$ radical solution were left in incubation for 2 hours in the dark. The control solution was prepared using distilled water instead of the sample. Absorbance at $734 \mathrm{~nm}$ wavelength was measured in a spectrophotometer. In the calculations, ABTS\% radical scavenging effect was found with the formula below.

$$
\begin{array}{r}
\text { ABTS scavenging }(\%)=\left[\begin{array}{r}
\left.\left(A_{\text {control }}-A_{\text {sample }}\right) / A_{\text {control }}\right] x \\
100
\end{array} \quad(\text { Eq. } 2)\right.
\end{array}
$$

Here, $A_{\text {control }}$ shows the absorbance of the control (ABTS solution without sample) and $A_{\text {sample shows }}$ the absorbance of the test sample.

\section{RESULTS AND DISCUSSION}

\section{UV Analysis}

The typical UV spectrum chart of the product obtained as a result of our study is shown in Figure 1. Accordingly, it was observed that the peak belonging to gallic acid at higher laccase concentrations than the two different laccase concentrations applied weakened as in the references and slightly shifted towards the red region. The protein absorption band at $280 \mathrm{~nm}$ was seen as a small shoulder of the dense absorption band of gallic acid (at $258 \mathrm{~nm}$ ). This shoulder peak may mean conjugation has occurred. BSA in this shoulder heteroconjugation is thought to belong to the amino acid tyrosine. That a similar shoulder peak at $280 \mathrm{~nm}$ was obtained in conjugation with myoglobin and gallic acid. However, it is difficult to evaluate the extent of complex formation using the density and shear properties of these signals (22). The weaker band at $280 \mathrm{~nm}$ suggests that conjugation is hetero-conjugation. This suggests that the amount of laccase present in the medium is not sufficient for homo-conjugation. These results are also supported by the references disclosed. In addition to these, when we examined the prepared samples, no precipitation was observed in the samples prepared at the ratio of BSA: GA (3: 1), while the samples prepared at the ratio of BSA: GA (1: 1) or BSA: GA (1: 3) were observed. It has been observed that the collapse occurs as the amount of BSA decreases. As a result of the reaction, gallic acid, which is oxidized and converted to quinones, binds to tyrosine residues in BSA.

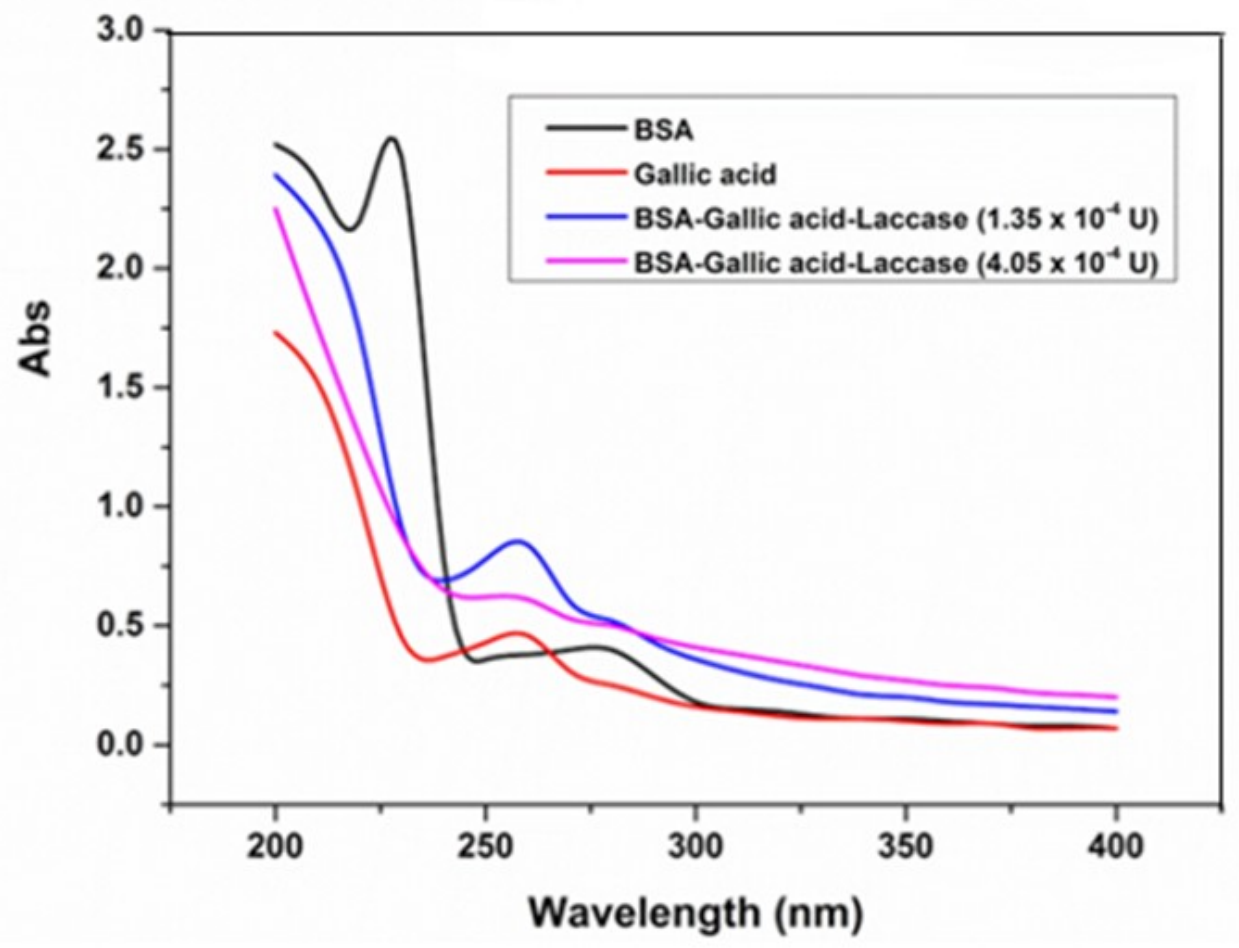

Figure 1: UV spectra of BSA, Gallic acid, and conjugates.

\section{FTIR Analysis}

The conjugation of gallic acid with BSA was analyzed by infrared spectroscopy. Polyphenolic characteristics with the existence of a phenolic ring
$-\mathrm{OH}$ stretching within the $3200-3550 \mathrm{~cm}^{-1}$. Amide band, which is located between $1700 \mathrm{~cm}^{-1}$ and $1600 \mathrm{~cm}^{-1}$, is composed mainly (around 80\%) of the $\mathrm{C}=\mathrm{O}$ stretching vibration of the peptidic bond 
(23). The spectral alterations observed are due to changes in the intensity of the amide band, upon acid interactions with protein $\mathrm{C}-\mathrm{O}, \mathrm{C}-\mathrm{N}$ and $\mathrm{NH}$ groups (hydrophilic contacts). The amide peak was moved to a lower wavelength due to the decreased a-helix content of the protein conjugated with gallic acid.

In the protein-flavonoid conjugate study performed by Kim and Paulo, FT-IR microscopy was used to analyze the chemical bonds formed by the conjugate. The study was carried out with a-casein and BSA proteins. Different peak formation was not observed in the conjugates of casein and BSA protein structures, and a change in the intensities of the peaks present in the protein was observed depending on the protein used (BSA, Casein) (19). Observation of the intensity decrease and shifts in the peaks belonging to the functional groups observed in the FT-IR analysis obtained in our study shows that the conjugate is formed.
In another study by Fan et al., bovine serum albumin (BSA)-caffeic acid (CA) conjugate was prepared by free radical-induced grafting method. In the study, FT-IR spectra of resveratrol, zein, BSA-CA conjugate and resveratrol loaded zeinBSA-CA nanoparticle, FT-IR spectroscopy were also used to analyze the intermolecular interactions between resveratrol and proteins. As a result of the analysis, zein protein and BSA-CA conjugate spectra were observed to be very similar and characteristic absorption bands were determined for both structures. It was stated that resveratrol did not cause new absorption peaks in the loaded zein-BSA-CA nanoparticle, indicating that the proteins and resveratrol were physically linked and did not exhibit any covalent reaction (24). The absence of an absorbance peak of the BSA-GA conjugate we obtained in our study is expressed by the fact that the interaction is not a covalent interaction as supported by the studies in the literature.

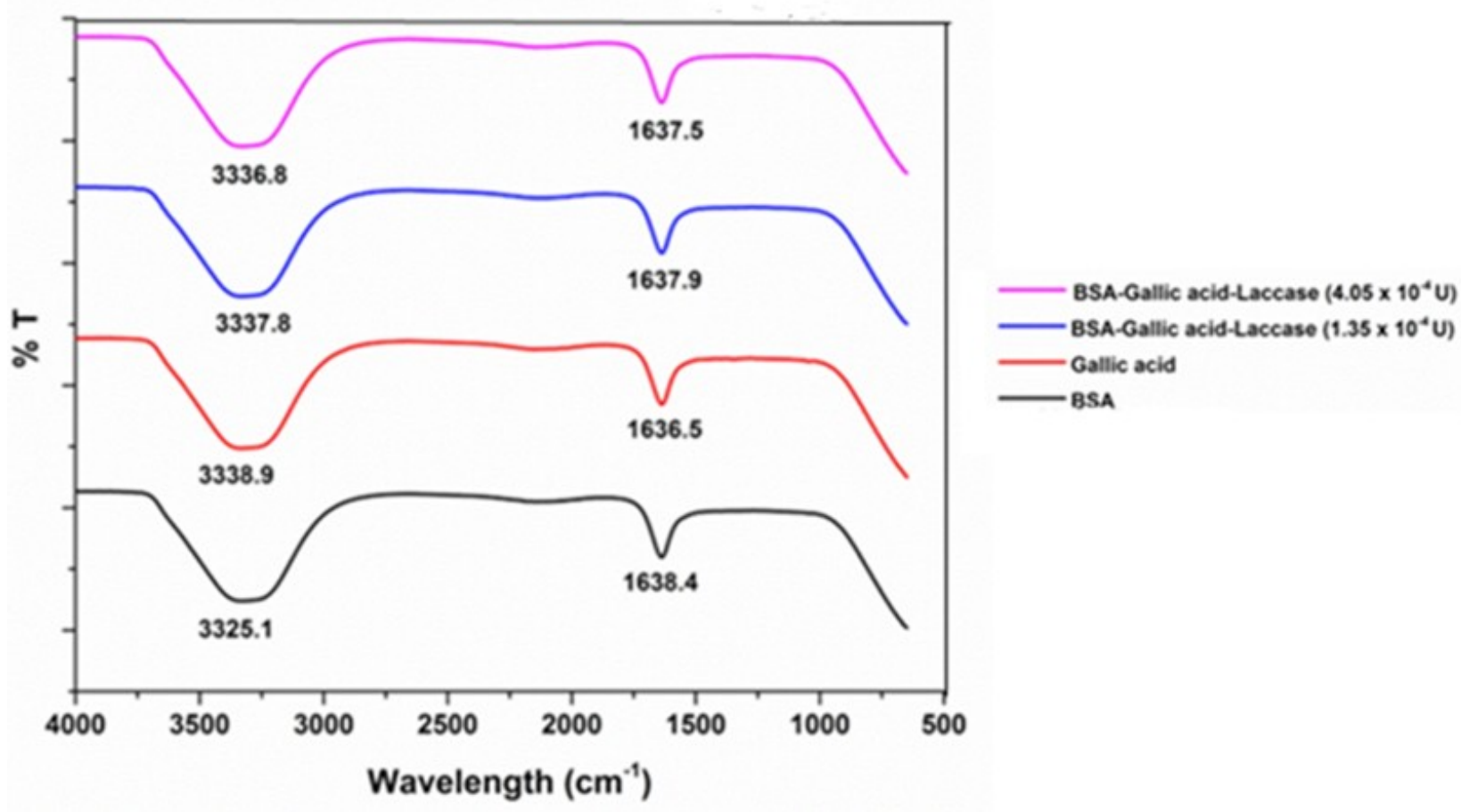

Figure 2: FTIR of BSA, Gallic acid, and conjugates.

\section{SDS PAGE}

The molecular weight distributions of pure BSA, GA, and BSA-gallic acid conjugate catalyzed by laccase were performed by SDS-PAGE (Figure 3). BSA-GA 1 represent BSA-gallic acid-laccase (1.35 $\left.x 10^{-4} \mathrm{U}\right)$ and BSA-GA 2 represent BSA-gallic acidlaccase $\left(4.05 \times 10^{-4} \mathrm{U}\right)$. The band of gallic acid was hardly detected (line 2) due to its low molecular weight. When both gallic acid and laccase were added to the BSA solution, the band moved slower than that of BSA and appeared higher in the gel because of its high molecular weight. This showed that GA and BSA were bound in the enzymatic process. 


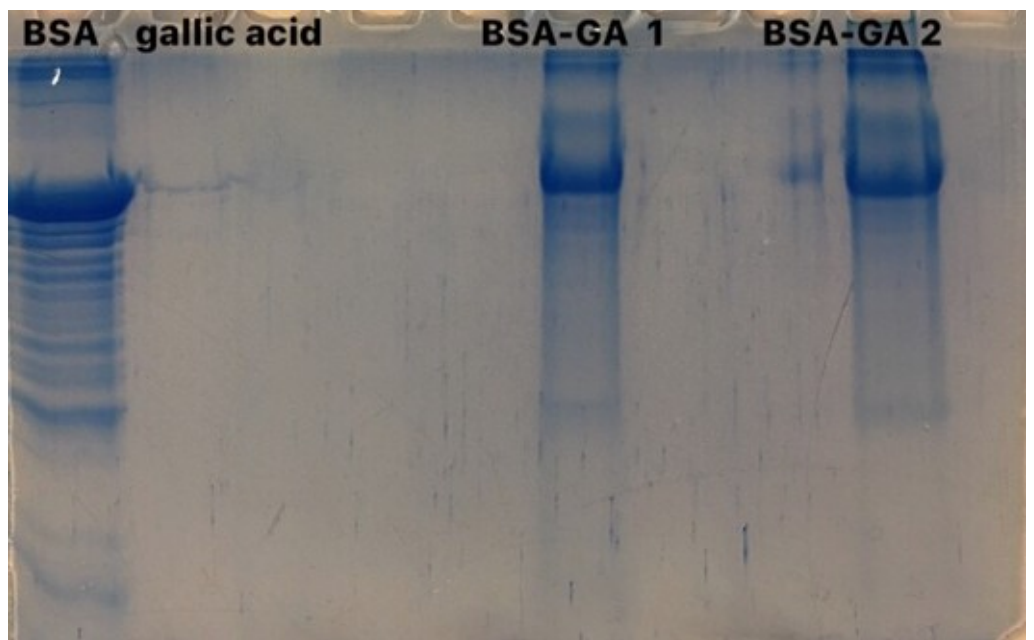

Figure 3: SDS-PAGE of BSA, GA, and conjugates.

\section{Antioxidant Activity}

In the DPPH test, the ability of BSA, GA, and conjugates to act as a donor for hydrogen atoms or electrons to reduce DPPH to DPPH-H was measured in a spectrophotometric manner. BSA does not have a DPPH radical scavenging activity. On the other hand, GA shows $94.53 \%$ radical inhibition activity. The BSA-GA conjugates formed as a result of the reaction show antioxidant activity. Conjugates showed an inhibitory activity of $86.79 \%$ and $81.96 \%$, respectively, depending on the excess amount of enzyme (Figure 4). Excessive amount of enzyme causes more GA-BSA interaction, thus increasing the activity.
In the ABTS test, the reaction between $A B T S$ and potassium persulfate forms the ABTS radical cation $\left(\mathrm{ABTS}^{+}\right.$) and a blue-green color is observed. When the antioxidant is present, the radical reverts to a colorless state. While gallic acid shows $96.57 \%$ ABTS radical inhibition activity, BSA does not show an antioxidant activity. The BSA-gallic acid conjugates showed a radical inhibition activity of $84.73 \%$ and $82.67 \%$ depending on the excess amount of enzyme (Figure 4). As in DPPH radical scavenging, the more enzyme amount increases the activity because it causes more gallic acid BSA interaction.

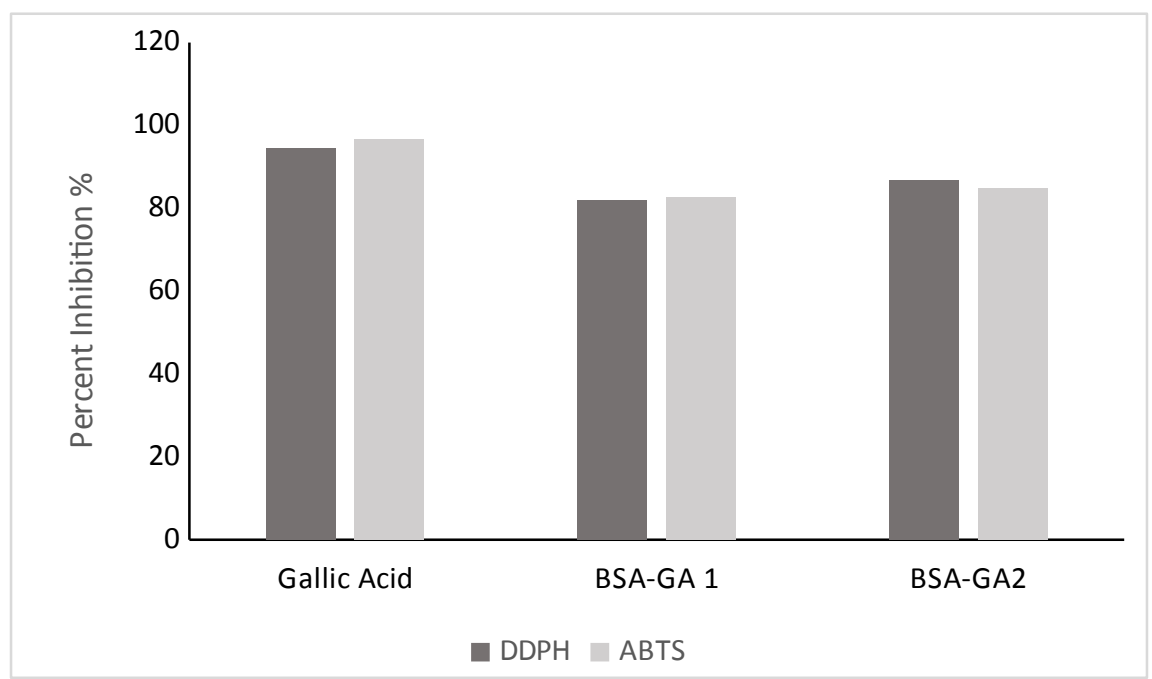

Figure 4: Antioxidant activity of Gallic acid, BSA-GA 1, BSA-GA 2.

\section{CONCLUSION}

In the present study, BSA was successfully functionalized with GA, a natural antioxidant, by laccase. The formation of BSA- gallic acid was proved by UV-Vis and FTIR spectra. Importantly, BSA-GA exhibited potent ABTS and DPPH scavenging activities, all of which were stronger than those of BSA. Protein-flavonoid conjugates can improve the natural properties of proteins, being promising products to be used in medical, food and polymer fields where antioxidant ability is an essential feature. 


\section{CONFLICT OF INTEREST}

The authors declare that they have no conflict of interest.

\section{REFERENCES}

1. Ls R, Nja S. Anticancer Properties of Phenolic Acids in Colon Cancer - A Review. J Nutr Food Sci [Internet]. 2016 [cited 2021 Nov 29];06(02). $<$ DOI $>$.

2. Weng C-J, Yen G-C. Chemopreventive effects of dietary phytochemicals against cancer invasion and metastasis: Phenolic acids, monophenol, polyphenol, and their derivatives. Cancer Treatment Reviews. 2012 Feb;38(1):76-87. $<$ DOI $>$.

3. Tian R-R, Pan Q-H, Zhan J-C, Li J-M, Wan S-B, Zhang $\mathrm{Q}-\mathrm{H}$, et al. Comparison of Phenolic Acids and Flavan-3-ols During Wine Fermentation of Grapes with Different Harvest Times. Molecules. 2009 Feb 18;14(2):827-38. <DOI $>$.

4. Badhani B, Sharma N, Kakkar R. Gallic acid: a versatile antioxidant with promising therapeutic and industrial applications. RSC Adv. 2015;5(35):27540-57. <DOI $>$.

5. Sagdicoglu Celep AG, Demirkaya A, Solak EK. Antioxidant and anticancer activities of gallic acid loaded sodium alginate microspheres on colon cancer. Current Applied Physics. 2020 Jul;S1567173920301164. <DOI>.

6. de Cristo Soares Alves A, Mainardes RM, Khalil NM. Nanoencapsulation of gallic acid and evaluation of its cytotoxicity and antioxidant activity. Materials Science and Engineering: C. 2016 Mar;60:126-34. <DOI $>$.

7. Giardina P, Faraco V, Pezzella C, Piscitelli A, Vanhulle S, Sannia G. Laccases: a never-ending story. Cell Mol Life Sci. 2010 Feb;67(3):369-85. $\leq$ DOI $>$.

8. Su J, Noro J, Fu J, Wang Q, Silva C, CavacoPaulo A. Enzymatic polymerization of catechol under high-pressure homogenization for the green coloration of textiles. Journal of Cleaner Production. 2018 Nov;202:792-8. <DOI>.

9. Su J, Castro TG, Noro J, Fu J, Wang Q, Silva C, et al. The effect of high-energy environments on the structure of laccase-polymerized poly(catechol). Ultrasonics Sonochemistry. 2018 Nov;48:275-80. <DOI $>$.

10. Jiang $Z$, Yuan $X$, Yao K, Li X, Zhang X, Mu Z, et al. Laccase-aided modification: Effects on structure, gel properties and antioxidant activities of a-lactalbumin. LWT. 2017 Jul;80:355-63. $<$ DOI $>$.

11. Aljawish A, Chevalot I, Jasniewski J, Paris C, Scher J, Muniglia L. Laccase-catalysed oxidation of ferulic acid and ethyl ferulate in aqueous medium: A green procedure for the synthesis of new compounds. Food Chemistry. 2014 Feb;145:104654. $\leq \mathrm{DOI}>$.

12. Mattinen $M-L$, Hellman $M$, Permi $P$, Autio $K$, Kalkkinen N, Buchert J. Effect of Protein Structure on Laccase-Catalyzed Protein Oligomerization. J Agric Food Chem. 2006 Nov 1;54(23):8883-90. $<$ DOI $>$.

13. Livney YD. Milk proteins as vehicles for bioactives. Current Opinion in Colloid \& Interface Science. 2010 Apr;15(1-2):73-83. <DOI $>$.

14. Matsudomi N, Rector D, Kinsella JE. Gelation of bovine serum albumin and $\beta$-lactoglobulin; effects of $\mathrm{pH}$, salts and thiol reagents. Food Chemistry. 1991 Jan;40(1):55-69. <DOI .

15. Božič M, Gorgieva S, Kokol V. Laccasemediated functionalization of chitosan by caffeic and gallic acids for modulating antioxidant and antimicrobial properties. Carbohydrate Polymers. 2012 Mar;87(4):2388-98. <DOI .

16. Yu C, Liu X, Pei J, Wang Y. Grafting of laccasecatalysed oxidation of butyl paraben and $p$ coumaric acid onto chitosan to improve its antioxidant and antibacterial activities. Reactive and Functional Polymers. 2020 Apr;149:104511. $\leq$ DOI $>$.

17. Božič M, Štrancar J, Kokol V. Laccase-initiated reaction between phenolic acids and chitosan. Reactive and Functional Polymers. 2013 Oct;73(10):1377-83. <DOI $>$.

18. Wolfenden BS, Willson RobinL. Radical-cations as reference chromogens in kinetic studies of onoelectron transfer reactions: pulse radiolysis studies of 2,2'-azinobis-(3-ethylbenzthiazoline-6sulphonate). J Chem Soc, Perkin Trans 2. 1982; (7):805-12. $\leq \mathrm{DOI}>$.

19. Kim S, Cavaco-Paulo A. Laccase-catalysed protein-flavonoid conjugates for flax fibre modification. Appl Microbiol Biotechnol. 2012 Jan;93(2):585-600. <DOI $>$.

20. Brand-Williams W, Cuvelier ME, Berset C. Use of a free radical method to evaluate antioxidant activity. LWT - Food Science and Technology. 1995;28(1):25-30. <DOI . 
21. Arnao MB, Cano A, Alcolea JF, Acosta M. Estimation of free radical-quenching activity of leaf pigment extracts. Phytochem Anal. 2001 Mar;12(2):138-43. <DOI .

22. Grigoryan KR, Shilajyan HA. Analysis of the interaction of gallic acid and myoglobin by UV-vis absorption spectroscopy. Russ J Bioorg Chem. 2017 May;43(3):255-8. <DOI>.
23. Grdadolnik J. Saturation effects in FTIR spectroscopy: intensity of amide I and amide II bands in protein spectra. Acta chimica slovenica. 2003;50(4):777-88.

24. Fan Y, Liu Y, Gao L, Zhang Y, Yi J. Improved chemical stability and cellular antioxidant activity of resveratrol in zein nanoparticle with bovine serum albumin-caffeic acid conjugate. Food Chemistry. 2018 Sep;261:283-91. <DOI>. 
Bat-Özmatara M, Ertan F. JOTCSA. 2022; 9(1): 29-36.

RESEARCH ARTICLE 